\title{
Optical conductivity of the infinite-dimensional Hubbard model
}

\author{
M. Jarrell ${ }^{1}$, J. K. Freericks ${ }^{2}$ and Th. Pruschke ${ }^{3}$ \\ ${ }^{1}$ Department of Physics, University of Cincinnati, Cincinnati, Ohio \\ ${ }^{2}$ Department of Physics, Georgetown University, Washington, DC 20057 \\ ${ }^{3}$ Institut für Theoretische Physik, Universität Regensburg, 93040 Regensburg, Germany
}

(December 7, 2017)

\begin{abstract}
A Monte Carlo-maximum entropy calculation of the optical conductivity of the infinite-dimensional Hubbard model is presented. We show that the optical conductivity displays the anomalies found in the cuprate superconductors, including a Drude width which grows linearly with temperature, a Drude weight which grows linearly with doping, and a temperature and doping-dependent mid-IR peak. These anomalies arise as a consequence of the dynamical generation of a quasiparticle band at the Fermi energy as $T \rightarrow 0$, and are a generic property of the strongly correlated Hubbard model in all dimensions greater than one.
\end{abstract}

Typeset using REVTEX

(C) 1994 by the authors. Reproduction of this article by any means is permitted for non-commercial purposes. 


\section{INTRODUCTION}

The discovery of high- $\mathrm{T}_{c}$ superconductors based on $\mathrm{CuO}$-compounds 1 has led to a large amount of theoretical work about the peculiar properties of these materials. A major effort has focussed on the normal-state properties of these compounds. This research was largely motivated and substantiated by experiments that revealed striking anomalies in the normal-state properties2. Most prominent among these are the linear resistivity, a linear NMR-relaxation of the Cu-spins, and a Hall angle that behaves $\sim T^{2}$ over a rather wide temperature region. Furthermore, the optical conductivity shows a Drude peak with a width B. $_{1} 1 / \tau \sim T$, consistent with the linearity of the resistivity, and a Drude weight which grows linearly with doping, consistent with the notion of holes acting as the charge carriers. In addition, there is a pronounced temperature and doping dependent mid-IR peak at frequencies above the Drude peak.

It was argued from the beginning that most of these anomalous properties can be explained by two special features appearing simultaneously in these materials: (i) They are strongly correlated, i.e. their (effective) local Coulomb interaction is comparable to or larger than the characteristic kinetic energy of the relevant carriers; and (ii) they are highly anisotropic with the electrons being in principle confined to the $\mathrm{CuO}$-planes characteristic for these compounds. Furthermore, it has been argued that the $\mathrm{CuO}$ planes can be accurately

described by a planar single-band Hubbard model 6 . The great interest in this class of materials has led to a number of new theoretical conjectures, that, although based on the assumption of strongly correlated carriers, focussed mainly on the 2D-character of the $\mathrm{CuO}$ planest 10 .

In two earlier publications11, we found that several of these anomalies can be understood from a Kondo-like effect in the infinite-dimensional Hubbard model. In particular, the density of states develops a sharp peak at the Fermi surface as the temperature is lowered. The development of this quasiparticle peak coincides with the screening of the effective local moments and anomalies in the transport. For example, the resistivity of the model displays a 
distinct linear in $T$ behavior with a slope that increases in inverse proportion to the doping $\delta$, consistent with experiment 12 . The NMR relaxation rate $1 / T_{1}$ displays a pronounced linear in $T$ region, with a slope that increases with doping, also consistent with experiment the qualitative features of the Hall resistivity are consistent with experiment2, including a Hall angle that increases quadratically with the temperature. Recently, a sharp Kondo-like peak at the Fermi surface has also been seen in the two-dimensional Hubbard model 13 as the temperature is lowered. This suggests that these anomalous normal-state properties are intrinsic to the Hubbard model, independent of dimensionality!

In this contribution, we address the anomalous normal-state properties of the Hubbardmodel optical conductivity. The optical conductivity $\sigma(\omega)$ is an important probe of the excitations of a strongly correlated system. It measures the rate at which electron-hole pairs are created by photons of frequency $\omega$. In a perfect (translationally invariant) metal, photons couple only to electron-hole pairs with vanishing momentum and energy; $\sigma(\omega)$ is proportional to a Dirac delta function $\left[\sigma(\omega)=D_{\text {perfect }} \delta(\omega)\right]$ with Drude weight $D_{\text {perfect }}=\pi e^{2} n / m$ (we set $\hbar=1)$. Electron-electron correlations modify this picture at zero temperature: The charge and spin fluctuations induce a dynamic disorder to the lattice potential which reduces the free-carrier Drude weight by the inverse of the quasiparticle renormalization factor $Z$ $\left(D=Z^{-1} D_{\text {perfect }}\right)$ and transfers the remaining spectral weight to a frequency-dependent component of $\sigma(\omega)$ reflecting the incoherent charge and spin fluctuations; the total spectral weight is, however, conserved. Finite-temperature effects will broaden the zero-frequency delta function into a Lorentzian and can modify both the quasiparticle renormalization and the higher-frequency excitations. This simple picture is further modified when restriction is made to a single (or finite number) of electronic bands. In this case, the total spectral weight can vary as a function of temperature or interaction strength because the projection onto a restricted basis set disregards all excitations to electronic bands that are higher in energy than those that have been kept in the model.

If the on-site Coulomb repulsion $U$ is large enough, then the system will be a Mott insulator at half-filling. The single-particle density of states $N(\omega)$ consists of two symmetric 
bands, separated by $U$ (called the lower and upper Hubbard bands) with the Fermi level lying in the middle of the gap. The renormalization factor $Z$ diverges since there are no quasiparticles at the Fermi level. The optical conductivity will consist of a charge-excitation peak centered at $\omega \approx U$, whose width is the order of the bandwidth. As the system is doped away from half filling, a quasiparticle resonance appears at the Fermi energy as $T \rightarrow 0$. The weight of the quasiparticle peak increases with doping. In this case, the optical conductivity will have a Drude peak (from the "free" quasiparticles at the Fermi energy) and a mid-IR band (because of excitations between the lower Hubbard band and the quasiparticle peak) in addition to the charge-excitation peak. The Drude weight can be estimated as follows: when $\delta$ holes are doped into the half-filled band, the total electron concentration is $(1-\delta)$, and the quasiparticle renormalization factor behaves roughly as $Z \propto 1 / \delta$, so the Drude weight is expected to be

$$
D_{\text {Drude }} \propto Z^{-1} n \propto \delta(1-\delta)
$$

which increases linearly for small and large doping, and is peaked near quarter filling ( $n=$ 0.5). In addition, the weight of the mid-IR peak should also behave qualitatively like Eq. (1) because it involves excitations between the quasiparticle peak and the lower Hubbard band, which also should grow as the density of holes in the half-filled band. The "hole-like" nature of the charge excitations near half filling results from the strong Coulomb renormalizations that create the Mott insulator at exactly half filling.

In this contribution, we investigate the optical conductivity of the Hubbard model in infinite dimensions. Our methodology is detailed in Section II, where we discuss the formalism and calculational techniques. Section III contains our results, which include a Drude peak whose width $1 / \tau$ grows linearly with temperature, a mid-IR peak that becomes more visible at low temperatures and dopings, and a charge-excitation peak that decreases with doping and is weakly dependent upon $T$. Comparison of our results with experiment is presented in Section IV, and conclusions are given in Section V. 


\section{METHOD}

Motivated by the observations of Anderson目 and Zhang and Ricel we will study the single-band Hubbard model 14 in d-dimensions, with

$$
H=-\frac{t^{*}}{2 \sqrt{d}} \sum_{<i j>\sigma} c_{i \sigma}^{\dagger} c_{j \sigma}+U \sum_{i} n_{i \uparrow} n_{i \downarrow}
$$

as a model for the $\mathrm{CuO}$ system. Our notation is the following: $c_{i \sigma}^{\dagger}$ is an electron creation operator for an electron in a localized state at lattice site $i$ with spin $\sigma ; n_{i \sigma}=c_{i \sigma}^{\dagger} c_{i \sigma}$ is the corresponding electron number operator; $t^{*}$ is the rescaled hopping matrix element; and $U$ is the Coulomb interaction strength. We choose $t^{*}=1$ as a convenient unit of energy. This model may be solved in the limit of high dimensions, using the observations of Metzner and Vollhardt 15 that with increasing coordination number of the underlying lattice the manybody renormalizations due to a two-particle interaction like the Hubbard- $U$ in (2) become purely local.

The solution of the model (2) may be mapped onto the solution of purely local correlated system coupled to an effective (self-consistently determined) bath 16 21. The quantum Monte Carlo (QMC) scheme based on the work of Hirsch and Fye20.22.23 has proven to be the most effective method for solving this strongly correlated local problem. Dynamical properties of the model are then obtained using numerical analytic continuation (employing maximumentropy techniques)24. This method requires a default model. To obtain the single-particle density of states we use the finite- $\mathrm{U}$ non-crossing approximation (NCA) 25 result for the infinite-dimensional Hubbard model as a default model at high temperatures, where it is essentially exact26. At low temperatures, where the NCA fails, we use the numerically continued result of the next higher temperature as a default model. The posterior probability of the final result is employed to determine which default model should be used. Generally, we find that the crossover temperature between using the NCA default model and the higher-temperature continuation lies at $T \approx 2 T_{0}$, where $T_{0}$ is the Kondo-like scale for this

model 11 . Once the density of states is determined, the self energy $\Sigma(\omega)$ may then be found 
by (numerically) inverting the Fadeev function $w(z)$ in the relation

$$
N(\omega)=\operatorname{Re}\{w[\omega+\mu-\Sigma(w)]\} / \sqrt{\pi} .
$$

With the knowledge of the one-particle self energy, one can calculate transport quantities. For example, the optical conductivity can be calculated exactly in the local approximation. It is given by the simple bubble only 26,27, whose evaluation leads to

$$
\begin{aligned}
\sigma_{x x}(\omega) & =\frac{e^{2} \pi}{V} \int_{-\infty}^{\infty} d \epsilon \frac{f(\epsilon)-f(\epsilon+\omega)}{\omega} \frac{1}{N} \sum_{\vec{k} \sigma}\left(\frac{\partial \epsilon_{\vec{k}}}{\partial k_{x}}\right)^{2} A\left(\epsilon_{\vec{k}}, \epsilon\right) A\left(\epsilon_{\vec{k}}, \epsilon+\omega\right) \\
& =\alpha \pi \int_{-\infty}^{\infty} d \epsilon \frac{f(\epsilon)-f(\epsilon+\omega)}{\omega} \int_{-\infty}^{\infty} d y \rho(y) A(y, \epsilon) A(y, \epsilon+\omega),
\end{aligned}
$$

where $V$ is the lattice volume, $\alpha=e^{2} /(V d)$ and defines the unit of the conductivity, the spectral weight satisfies $A\left(\epsilon_{k}, \omega\right)=-\frac{1}{\pi} \operatorname{Im}[G(k, \omega)]$, and the noninteracting density of states is $\rho(y)=\exp \left(-y^{2}\right) / \sqrt{\pi}$.

As the temperature is lowered, the Hubbard model in infinite-d is found to always be a Fermi liquid20,26, except for the region of phase space where it is magnetices. A Fermi liquid is defined by a self-energy that has the following structure:

$$
\begin{aligned}
& \operatorname{Re} \Sigma\left(\omega+i 0^{+}\right)=\operatorname{Re} \Sigma(0)+\omega(1-Z)+O\left(\omega^{2}\right), \\
& \operatorname{Im} \Sigma\left(\omega+i 0^{+}\right)=-\Gamma+O\left(\omega^{2}\right),
\end{aligned}
$$

with $\Gamma \propto T^{2}$ for temperatures $T \ll T_{0}$ the characteristic Fermi temperature. [The Fermi temperature $T_{0}$ decreases to zero as half filling is approached 11 , and the Fermi-liquid-theory form of Eq. (5) still holds for moderate temperatures, with the only change being $\Gamma \propto T$ for $T>T_{0}$.] The spectral weight, then assumes the form

$$
A(y, \omega)=\frac{1}{\pi} \frac{\Gamma}{\Gamma^{2}+\left(\omega Z+\epsilon_{F}-y\right)^{2}}+A_{\text {Inc }}(y, \omega)
$$

with the Fermi level defined by $\epsilon_{F} \equiv \mu-\operatorname{Re} \Sigma(0)$ and $A_{I n c}(y, \omega)$ denoting the (rather structureless) incoherent contributions to the spectral function. The spectral function includes a delta function at zero temperature $\left[A(y, \omega) \rightarrow \delta\left(\omega Z+\epsilon_{F}-y\right)+A_{\text {Inc }}(y, \omega)\right]$ because the broadening $\Gamma$ vanishes in that limit. 
If the Lorentzian form for the Fermi-liquid-theory spectral function [Eq. (6)] is substituted into the expression for the optical conductivity found in Eq. (伪), and the temperature satisfies $T \ll T_{0} \ll t^{*}$, then the optical conductivity becomes

$$
\frac{\sigma(\omega)}{\alpha}=\frac{D}{\pi} \frac{\tau}{1+\omega^{2} \tau^{2}}+\frac{\sigma_{I n c}(\omega)}{\alpha}
$$

with Drude weight $D=Z^{-1} \pi \rho\left(\epsilon_{F}\right)$, relaxation time $\tau=1 /(2 \Gamma) \propto 1 / T^{2}$, and $\sigma_{\text {Inc }}(\omega)$ containing the contributions from the incoherent pieces of the spectrum.

The noninteracting Drude weight for the single-band model $(Z=1)$ satisfies $D_{\text {non }}=$ $\pi \rho\left(\epsilon_{F}\right)$. As the interaction $U$ is turned on in a single-band model, the total integrated spectral weight is not conserved but becomes $U$-dependent 29 because spectral weight that would be shifted to higher bands is "lost" in any single-band model. The total spectral weight satisfies

$$
2 \int_{0}^{\infty} \sigma(\omega) d \omega=-\pi e^{2}\left\langle T_{x}\right\rangle
$$

where $\left\langle-T_{x}\right\rangle$ is the kinetic energy per site, divided by the number of lattice dimensions. It should be stressed that the modification of the sum rule in Eq. (\$) will produce some systematic modifications to the behavior of the optical conductivity as a function of temperature, interaction strength, and doping. These systematic effects must be kept in mind when one is comparing the results of a single-band calculation to experiment.

The Drude weight $D$ for the interacting system, may also be determined (at $T=0$ ) by extrapolation of the Matsubara-frequency current-current correlation function using the method proposed by Scalapino et al 30 ( $D$ measures the "free" quasiparticles in the system). This method determines the Drude weight of a metal by examining the asymptotic form of the current-current susceptibility in the x-direction, $\Lambda_{x x}\left(\mathbf{q}, i \omega_{n}\right)$. More specifically, $D$ is given by

$$
D=\lim _{T \rightarrow 0} \pi\left[e^{2}\left\langle-T_{x}\right\rangle-\Lambda_{x x}(\mathbf{q}=0,2 i \pi T)\right]
$$

where the limit $T \rightarrow 0$ is taken after first setting the momentum transfer to zero $(\mathbf{q}=0)$. Note that this latter method of determining the Drude weight is a much better defined 
procedure than trying to fit the optical conductivity to the generic form of Eq. (অ) because of the uncertainty left in trying to fit $\sigma_{\text {Inc }}(\omega)$.

\section{RESULTS}

We present here our results for the optical conductivity of the single-band Hubbard model in infinite dimensions with $U=4 t^{*}$. Figure 11(a) shows the optical conductivity obtained from Eq. (4) when $\delta=0.068$ for a variety of temperatures. One finds the Drude peak at $\omega=0$ developing with decreasing temperature. In addition, a shoulder develops adjacent to the Drude peak at $\omega \approx 1$ which is strongly temperature dependent and clearly visible only for the lowest temperatures. The last feature in $\sigma(\omega)$ is a roughly temperature-independent peak at $\omega \approx U$. In order to compare our results to experiment, the three features in $\sigma(\omega)$ are fit to a Lorentzian plus (asymmetric) harmonic-oscillator forms for the higher-energy peaks

$$
\frac{\sigma(\omega)}{\alpha} \approx \frac{D}{\pi} \frac{\tau}{1+\omega^{2} \tau^{2}}+\frac{C_{M I R}}{\pi} \frac{\omega^{2} \Gamma_{M I R}}{\omega^{2} \Gamma_{M I R}^{2}+\left(\omega^{2}-\omega_{M I R}^{2}\right)^{2}}+\frac{C_{C}}{\pi} \frac{\omega^{2} \Gamma_{C}}{\omega^{2} \Gamma_{C}^{2}+\left(\omega^{2}-\omega_{C}^{2}\right)^{2}},
$$

with $\tau$ the relaxation time of the quasiparticles, and the constants $C_{M I R}, \omega_{M I R}$, and $\Gamma_{M I R}$ $\left(C_{C}, \omega_{C}\right.$, and $\left.\Gamma_{C}\right)$ the weight, center, and width, respectively of the mid-IR (charge-transfer) peak. The Drude width $1 / \tau$ obtained from this fitting procedure is plotted in the inset to Fig. 1(b). Note that for temperatures on the order of $T_{0}$, it is well approximated by a straight line (the line is a guide to the eye), while for $T \ll T_{0}$ the Drude width must change its behavior to $\tau \propto T^{2}$ according to the general properties of a Fermi liquid. By subtracting off the fit Drude portion from the optical conductivity, we were able to isolate the mid-IR portion, as shown in Fig. 1(b). Note that the "double-peak" structure emerging in the mid-IR peak at the lowest temperature is an artifact of the fitting procedure which is not perfect in extracting the parameters for the Drude peak. The mid-IR peak is temperature dependent, growing in size and moving to slightly lower frequencies as the temperature is lowered.

Comparison of the Drude weight $D$ determined by the fitting procedure in Eq. (10) and the independent method of calculation in Eq. (9) produces only qualitative agreement. 
We have tried more sophisticated fitting routines in which we fit the Fermi-liquid-theory parameters $\left(Z, \Gamma\right.$, and $\left.\epsilon_{F}\right)$ in the spectral function of Eq. (6) and then determine the Drude contribution to the optical conductivity by employing the full expression in Eq. (4), and the fit improves, as does the comparison of the Drude weight, but the quantitative agreement still has a systematic error on the order of $10-20 \%$ (where the fitting procedure always overestimates the Drude weight), because of the simple form chosen for the mid-IR peak. Our conclusion is that the method of Ref. 30 is superior to any ad hoc fitting procedure in determining the low-temperature Drude weight. Note, however, that the charge-transfer peak is uniquely determined by the above fitting procedure (in the sense that the results are independent of what fitting procedure is employed), indicating that the harmonic-oscillator form is a reasonable approximation to that peak.

The existence of the Drude and charge-excitation peaks have been reported previouslyet. These results were obtained with the NCA and were thus restricted to temperatures $T \gtrsim 2 T_{0}$. The mid-IR bump, however, becomes clearly visible only for temperatures below this scale. We can presently resolve this feature because of the refined numerics employed in the QMC and the numerical analytic continuation at lower temperatures.

In Fig. Q, the optical conductivity is plotted as a function of doping for fixed temperature $\beta=43.2$. The different dopings are best identified by their decreasing charge-transfer peaks $(\delta=0.068,0.0928,0.1358,0.1878,0.2455,0.3,0.35,0.4$, and 0.45$)$. The solid lines indicate the electron concentrations where the optical conductivity increases with doping in the lowerHubbard-band region of $\omega<2(\delta<0.25)$ and the dotted lines are where $\sigma(\omega)$ decreases with doping in the same region $(\delta>0.25)$. One can see that the mid-IR peak is strongly doping dependent, being most distinct from the Drude peak at low dopings, and merging with it as the doping and the width of the Drude peak increases. In the inset, we show that the Drude weight $D$ increases linearly with $\delta$. This latter result shows that the "free" carriers in a doped Mott insulator are holes in the half-filled band. As the doping is increased further, the Drude weight eventually saturates, and then decreases with doping, as the character of the "free" carriers changes from being hole-like to being electron-like. 
The optical conductivity clearly displays an isobestic point, where $\sigma(\omega)$ is independent of doping $\left(\omega_{I B} \approx 2\right)$. The isobestic point marks the boundary between the regions where the weight of the optical conductivity increases as a function of doping $\left(\omega<\omega_{I B}\right)$, and the regions where the weight decreases with doping $\left(\omega>\omega_{I B}\right)$. However, it is incorrect to assume that all of the spectral weight that lies below the isobestic point was transferred from above. An effective carrier number $N_{e f f}(\omega)$, defined by the integral of the optical conductivity

$$
N_{e f f}(\omega)=\frac{2}{\pi} \int_{0}^{\omega} \frac{\sigma(\Omega)}{\alpha} d \Omega \quad,
$$

is plotted in Figure 3 (the normalization is chosen here to give the number of carriers for a model with a complete set of basis states in order to make contact with experiment). The integrated optical conductivity increases rapidly with doping for small values of $\delta$, but then increases more slowly, eventually saturating, and decreasing with doping (at $\delta \approx 0.4$ ). Once again, solid lines denote the electron concentrations where $N_{\text {eff }}(\omega)$ increases with doping $(\delta<0.4)$ and the dotted line is where $N_{\text {eff }}(\omega)$ decreases with doping $(\delta>0.4)$. Note that there is no frequency where the integrated spectral weight is independent of doping, which would be required for the above scenario (of the Drude and mid-IR weight coming entirely from the charge-transfer peak) to occur.

In addition, we can examine how the sum rule for the total spectral weight, and for it's component pieces (Drude, mid-IR, and charge-transfer) evolve as a function of doping at fixed low temperature (in fact, the following analysis is an approximation to the zerotemperature behavior of the different components of the spectral weight). This is shown in Figure 4. The total spectral weight is found from Eq. (8) (which agrees with the integral of the optical conductivity to within $3 \%$ ), the charge-transfer weight from the fitting procedure in Eq. (10), the Drude weight from Eq. (9), and the weight of the mid-IR peak is then determined by subtraction (the charge-transfer weight is neglected for $n<0.5$ ). Note how the mid-IR peak initially increases linearly with doping until $n \approx 0.75$ where it saturates and then decreases as the system becomes less correlated, and how the system changes from a 
hole-like metal to an electron-like metal at about quarter filling. In addition, one can easily see that the Drude and mid-IR weights both grow quite rapidly as the system is doped away from half filling (the initial growth rate of the mid-IR weight is about a factor of 2 faster than that of the Drude weight). This fast growth of the Drude and mid-IR weights explains why the optical conductivity for $\omega<\omega_{I B}$ increases so rapidly with doping as shown in Fig. 2 . These results are qualitatively similar to those found in the strong-coupling limit of the Hubbard model in one dimension 3 , indicating that the optical properties of the Hubbard model are not strongly dependent upon the dimensionality (with the exception that there is no mid-IR peak in one dimension).

One can also inquire into the temperature dependence of the Drude, mid-IR, and chargetransfer peaks at fixed doping. The analysis, in this case, relies on trying to fit the optical conductivity to the form in Eq. 10, as a function of temperature. Such a procedure is ill-defined, because the Drude and mid-IR peaks merge at high temperatures, and it is difficult to separate them into their component pieces, without explicit knowledge of the proper fitting forms. However, the qualitative physics can be discussed: We find that as the temperature is raised, that spectral weight is lost from the system (i. e., the magnitude of the expectation value of the kinetic-energy operator decreases as $T$ increases), that the Drude and charge-transfer weights slightly increase, and that the mid-IR weight is sharply reduced. In addition, spectral weight rapidly shifts to higher frequency, since the Drude width (which is easy to determine by the fitting procedure) increases linearly with $T$ for temperatures larger than $T_{0}$.

The origin of all of these features in the optical conductivity can be understood by studying the single-particle density of states, plotted as a function of both temperature (a) and doping (b) in Fig. 5. Clearly the Drude peak results from the quasiparticle band which develops at low temperatures. We have shown previously that while this peak is developing (i. e. in the region $T>T_{0}$ ), the scattering rate (as measured by the resistivity) increases in proportion to the temperature 11 . Thus, the Drude width also increases in proportion to the temperature. We attribute the mid-IR peak to excitations from the lower Hubbard band to 
the quasiparticle band at the Fermi energy. Note that the quasiparticle resonance is only sharp and distinct from the lower Hubbard band at low $T$ and small $\delta$. Thus, the mid-IR peak will only be visible in the optical conductivity at low $T$ and small $\delta$ as well. On the other hand, the upper peak at $\omega \approx 4$ is due to the charge excitations from both the lower Hubbard band and the quasiparticle band to the upper Hubbard band. Since the upper Hubbard band is distinct from the quasiparticle weight at the Fermi surface, and since the weight in the upper Hubbard band does not change as significantly with doping, the upper peak in $\sigma(\omega)$ has a weaker temperature and doping dependence, but is expected to disappear as the system becomes uncorrelated in the low-density limit.

\section{COMPARISON TO EXPERIMENT}

Many experimental measurements have been made of the optical conductivity in doped cuprates. Kramers-Kronig analysis is used to determine $\sigma(\omega)$ from reflectivity measurements in $32 \mathrm{La}_{2-x} \mathrm{Sr}_{x} \mathrm{CuO}_{4}$ (LSCO), in $335 \mathrm{YBa}_{2} \mathrm{Cu}_{3} \mathrm{O}_{6+x}$ (YBCO), in $34 \mathrm{Bi}_{2} \mathrm{Sr}_{2} \mathrm{CaCu}_{2} \mathrm{O}_{x}$ (BSCCO), and in $36,35 \mathrm{Nd}_{2} \mathrm{CuO}_{4-y}(\mathrm{NCO})$; reviews have also been published 14 . Photoinduced absorption is also employed to measure the optical conductivity in 37 YBCO and in both 38 LSCO and NCO. It is interesting to compare the theoretical calculation of the optical conductivity with these experimental results.

The experiments yield six trends for the cuprate superconductors: 1) The mid-IR peak maximum moves to lower frequency, and merges with the Drude peak as the doping increases; its spectral weight grows very rapidly with doping near half filling; 2) At a fixed value of the doping, spectral weight rapidly moves to lower frequency as $T \rightarrow 0$, but the total weight in the Drude and mid-IR peaks remains approximately constant; the width of the Drude peak decreases linearly with $T$; 3) The insulating (or undoped) phase has a charge-transfer gap; when doped, the optical conductivity initially increases within the gap region, but eventually saturates and then decreases with doping; 4) There is an isobestic point or nearly isobestic behavior (in that the optical conductivity is nearly independent of doping) at a 
frequency that is approximately one half of the charge-transfer gap; 5) The effective charge has a constant value with respect to doping near the charge-transfer edge; and 6) more than one peak is observed within the mid-IR region.

Most of these trends are reproduced by our theoretical model. In particular, Fig. 2 illustrates how the mid-IR peak moves to lower frequency and joins the Drude peak (1), how the optical conductivity initially increases with doping at low frequency, but then saturates and decreases (3), and how there is an isobestic point (4). Fig. [ 1 shows how the spectral weight is transferred to lower frequencies as the temperature is lowered, but we find that the total (Drude plus mid-IR) spectral weight does not remain constant as in (2) because the temperature dependence of the expectation value $\left\langle T_{x}>\right.$ produces some temperature dependence to the total Drude plus mid-IR spectral weight (it actually decreases as $T$ increases). Fig. 固 does not display the trend of point (5), possibly because the restriction to a single-band model reduces the optical conductivity at higher frequencies to such an extent that the effective charge must depend on the doping level for the theoretical model. We do not see the multiple peaks in the mid-IR region of point (6).

These experimental features are usually attributed 39 to phonons or impurities present in the system, but judging from our results, the low-energy feature may also be due to excitations from the lower Hubbard band to a dynamically generated quasi-particle band at the Fermi energy (which is generated by Kondo-like screening of the moments). Naturally, the single-band model fits the experminental data much better at lower frequencies, where the single-band approximation is relevant, but fails in reproducing some of the higher-energy trends found in the cuprates, because of the neglected bands. A comprehensive theory should include both the effects of strong electron correlation (which reproduce the insulator at half filling, and give a hole-like character to the charge excitations near half filling) with the effect of electron-phonon coupling (which are necessary to explain similar mid-IR features in nonsuperconducting perovskites). 


\section{CONCLUSION}

A quantum Monte Carlo and maximum entropy calculation of the optical conductivity of the infinite-dimensional Hubbard model has been presented. The Mott-insulating character of the ground state at half filling drives many anomalous behaviors in the normal state near half filling that are similar to those observed in the cuprate superconductors. In particular the system is always a Fermi liquid away from half filling, but the Fermi temperature vanishes, and the quasiparticle renormalization factor diverges as half filling is approached. As a result, the free carriers in the system initially have a hole-like character (that changes to an electron-like character at approximately quarter filling). The Drude width for these carriers grows linearly with temperature for temperatures above $T_{0}$, the Drude weight grows linearly with doping, and there is a doping and temperature dependent mid-IR peak. These anomalies arise naturally from the presence of a strongly temperature-dependent quasiparticle peak, whose origin is a Kondo-like screening of the magnetic moments, and which appears to occur in the Hubbard model for all dimensions greater than 1.

The anomalous features in the experimentally measured optical conductivity for the cuprates are usually attributed to either polarons or impurities. However, any purely polaronic theory has difficulty in explaining the magnetic insulating character of the ground state at half filling. The Hubbard model naturally describes such an insulating state, and

appears to also describe many of the anomalous features present in the experimental data. In light of this fact, it is worthwhile to try to incorporate both the effects of strong electron correlation, and the electron-phonon interaction into a comprehensive theory for the normal state of the cuprate materials. Work along these lines is in progress.

\section{ACKNOWLEDGMENTS}

We would like to acknowledge useful conversations with W. Chung, J. Keller, Y. Kim, D. Scalapino, R. Scalettar, D. Tanner, and G. Thomas. This work was supported by the Na- 
tional Science Foundation grant number DMR-9107563, the NATO Collaborative Research Grant number CRG 931429 and through the NSF NYI program. In addition, we would like to thank the Ohio Supercomputing Center, and the physics department of the Ohio State University for providing computer facilities. 


\section{REFERENCES}

${ }^{1}$ J.G. Bednorz and K.A. Müller, Z. Phys. 64, 189 (1986).

${ }^{2}$ For reviews of relevant experiments see C. H. Pennington and C. P. Slichter, in Physical Properties of High Temperature Superconductors edited by D. M. Ginsberg Vol.2; N. P. Ong ibid.; Y. Iye ibid. Vol. 3.

${ }^{3}$ D.B. Romero, C. D. Porter, D. B. Tanner, L. Forro, D. Mandrus, L. Mihaly, G. L. Carr, and G. P. Williams, Phys. Rev. Lett. 68, 1590 (1992); see also L. Forro, G. L. Carr, G. P. Williams, D. Mandrus, and L. Mihaly, Phys. Rev. Lett. 65, 1941 (1990).

${ }^{4}$ For a review, please see T. Timusk and D. B. Tanner, in Physical Properties of High Temperature Superconductors, Vol. 1, edited by D. M. Ginsberg (World Scientific, Singapore, 1989), pp. 339-407, G. A. Thomas, in Proceedings from the 39th Scottish Universities Summer School in Physics, edited by D.P. Tunstall, W. Barford, and P. Osborne (Adam Hilger, New York, 1991), pp. 169-206, and references contained therein.

${ }^{5}$ P.W. Anderson, Frontiers and Borderlines in Many Particle Physics, Proceedings of the International School of Physics "Enrico Fermi" (North Holland, Amsterdam, 1987), p. 1; P.W. Anderson, Science 235, 1196 (1987).

${ }^{6}$ F. C. Zhang and T. M. Rice, Phys. Rev. B 37, 3759 (1988).

${ }^{7}$ G. Baskaran, P.W. Anderson, Phys. Rev. B37, 850 (1988).

${ }^{8}$ J. Sólyom, Adv. Phys. 28, 201 (1979).

${ }^{9}$ P.W. Anderson, Physica C 185, 11 (1991).

${ }^{10}$ C.M. Varma, P.B. Littlewood, S. Schmitt-Rink, E. Abrahams and A.E. Ruckenstein, Phys. Rev. Lett. 63, 1996 (1989).

${ }^{11}$ M. Jarrell and Th. Pruschke, Phys. Rev. B49, 1458 (1993); Th. Pruschke and M. Jarrell, Physica B 199\&200, 217(1994). 
${ }^{12}$ Christoph Quitmann, Ph.D. thesis, Aachen 1992 (unpublished).

${ }^{13}$ N. Bulut, D. J. Scalapino, and S. R. White, Phys. Rev. Lett. 72, 705 (1994).

${ }^{14}$ J. Hubbard, Proc. R. Soc. (London) A 276, 238 (1963); M.C. Gutzwiller, Phys. Rev. Lett. 10, 159 (1963); J. Kanamori, Prog. Theor. Phys. 30, 257 (1963).

${ }^{15}$ W. Metzner and D Vollhardt, Phys. Rev. Lett. 62, 324 (1989).

${ }^{16}$ U. Brandt and C. Mielsch, Z. Phys. B 75, 365 (1989); Z. Phys. B 79, 295 (1990); Z. Phys. B 82, 37 (1991).

${ }^{17}$ V. Janiš, Z. Phys. B 83, 227 (1991).

${ }^{18}$ C. Kim, Y. Kuramoto and T. Kasuya, J. Phys. Soc. Japan 59, 2414 (1990).

${ }^{19}$ V. Janiš and D. Vollhardt, Int. J. Mod. Phys. B6, 713 (1992).

${ }^{20}$ M. Jarrell Phys. Rev. Lett. 69, 168 (1992).

${ }^{21}$ A. Georges and G. Kotliar, Phys. Rev. B45, 6479 (1992).

${ }^{22}$ J.E. Hirsch and R.M. Fye, Phys. Rev. Lett. 56, 2521 (19 86).

${ }^{23}$ M. Jarrell, H. Akhlaghpour, and Thomas Pruschke, Quantum Monte Carlo Methods in Condensed Matter Physics, edited by M. Suzuki, (World Scientific, 1993).

${ }^{24}$ J.E. Gubernatis, M. Jarrell, R.N. Silver, and D.S. Sivia, Phys. Rev. B 44, 6011 (1991); and M. Jarrell and J.E. Gubernatis, to appear.

${ }^{25}$ H. Keiter and J.C. Kimball, Intern. J. Magnetism 1, 233(1971); N.E. Bickers, D.L. Cox and J.W. Wilkins, Phys. Rev. B36, 2036 (1987); Th. Pruschke and N. Grewe, Z. Phys. B 74, 439 (1989).

${ }^{26}$ Th. Pruschke, D.L. Cox and M. Jarrell, Phys. Rev. 47, 3553 (1993); Th. Pruschke, D.L. Cox and M. Jarrell, Europhys. Lett. 21 , 593 (1993). 
${ }^{27}$ A. Khurana, Phys. Rev. Lett. 64, 1990 (1990).

${ }^{28}$ J. K. Freericks and M. Jarrell, Phys. Rev. Lett. (to appear); P. G. J. van Dongen, (submitted to Phys. Rev. Lett.).

${ }^{29}$ P. F. Maldague, Phys. Rev. B 16, 2437 (1977).

${ }^{30}$ D.J. Scalapino, S. White and S. Zhang, Phys. Rev. B, 47, 7995 (1993).

${ }^{31}$ H. Eskes and A. M. Oleś, Phys. Rev. Lett. 73, 1279 (1994).

${ }^{32}$ S. Uchida, T. Ido, H. Takagi, T. Arima, Y. Tokura, S. Tajima, Phys. Rev. B 43, 7942 (1991); S. Uchida, J. Phys. Chem. Solids, 53, 1603 (1992).

${ }^{33}$ J. Orenstein, G. A. Thomas, A. J. Millis, S. L. Cooper, D. H. Rapkine, T. Timusk, L. F. Schneemeyer, and J. V. Waszczak, Phys. Rev. B 42, 6342 (1990).

${ }^{34}$ D.B. Romero, et al. (unpublished).

${ }^{35}$ G. A. Thomas, D. H. Rapkine, S. L. Cooper, S-W. Cheong, and A. S. Cooper, Phys. Rev. Lett. 67, 2906 (1991); G. A. Thomas, D. H. Rapkine, S. L. Cooper, S-W. Cheong, A. S. Cooper, L. F. Schneemeyer, and J. V. Waszczak, Phys. Rev. B 45, 2474 (1992).

${ }^{36}$ S. Uchida, H. Takagi, Y. Tokura, S. Koshihara, and T. Arima, in Strong Correlation and Superconductivity, edited by H. Fukuyama, S. Maekawa, and A. P. Malozemoff (Springer, Tokyo, 1989) pp. 194-203.

${ }^{37}$ G. Yu, C. H. Lee, D. Mihailovic, A. J. Heeger, C. Fincher, N. Herron, and E. M. McCarron, Phys. Rev. B 48, 7545 (1993).

${ }^{38}$ Y.H. Kim, S.-W. Cheong, and Z. Fisk, Phys. Rev. Lett. 67, 2227 (1991).

${ }^{39}$ A. S. Alexandrov, V. V. Kabanov, and D. K. Ray, Physica C 224, 247 (1994). 


\section{FIGURES}

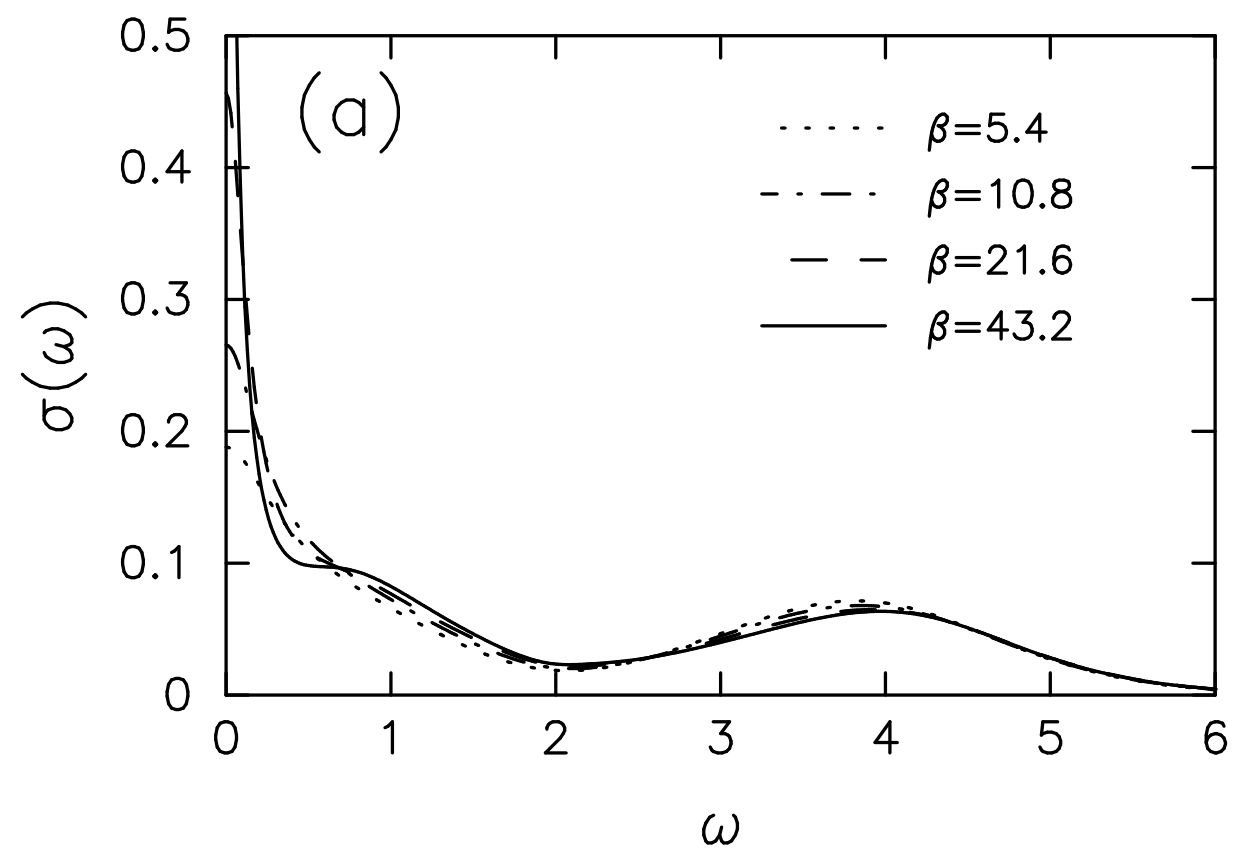

Jarrell, Freericks, and Pruschke, Phys. Rev. B, Figure 1(a)

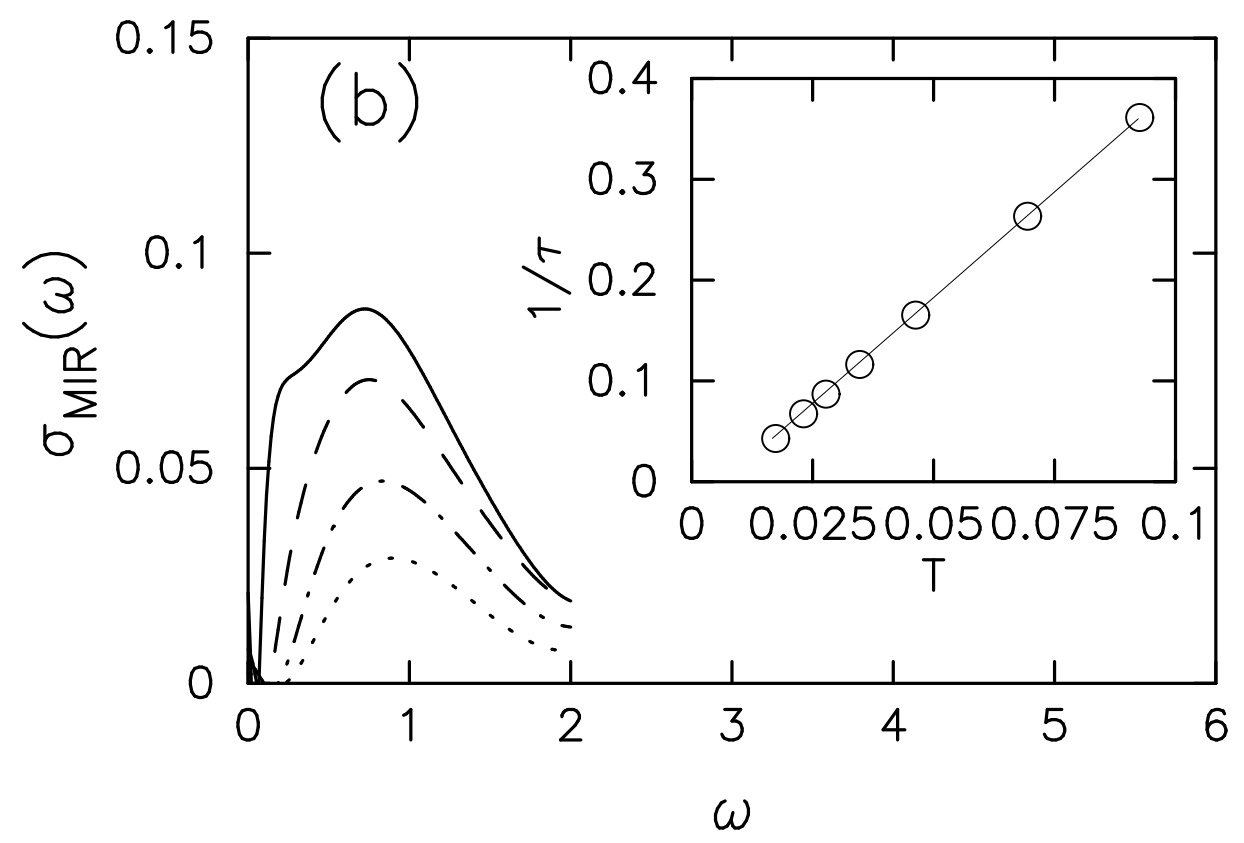

Jarrell, Freericks, and Pruschke, Phys. Rev. B, Figure 1(b) 
FIG. 1. (a) Optical conductivity vs. $\omega$ for various temperatures when $U=4$ and $\delta=0.068$ in units of $\alpha=e^{2} /(V d)$. Note that at low temperatures, when the Kondo peak becomes pronounced in the DOS (see Fig. 5), a mid-IR feature begins to appear in $\sigma(\omega)$. The mid-IR feature is isolated in (b) by fitting the low-frequency data with Eq. 10, and subtracting off the Drude part. As the temperature is lowered, the mid-IR peak becomes more pronounced and shifts to lower temperatures. Note that the double-peak structure in the mid-IR peak is most likely due to the inaccurate fitting form for $\sigma_{M I R}(\omega)$. As shown in the inset, the width $(1 / \tau)$ of the Drude peak is found to increase roughly linearly with $T$.

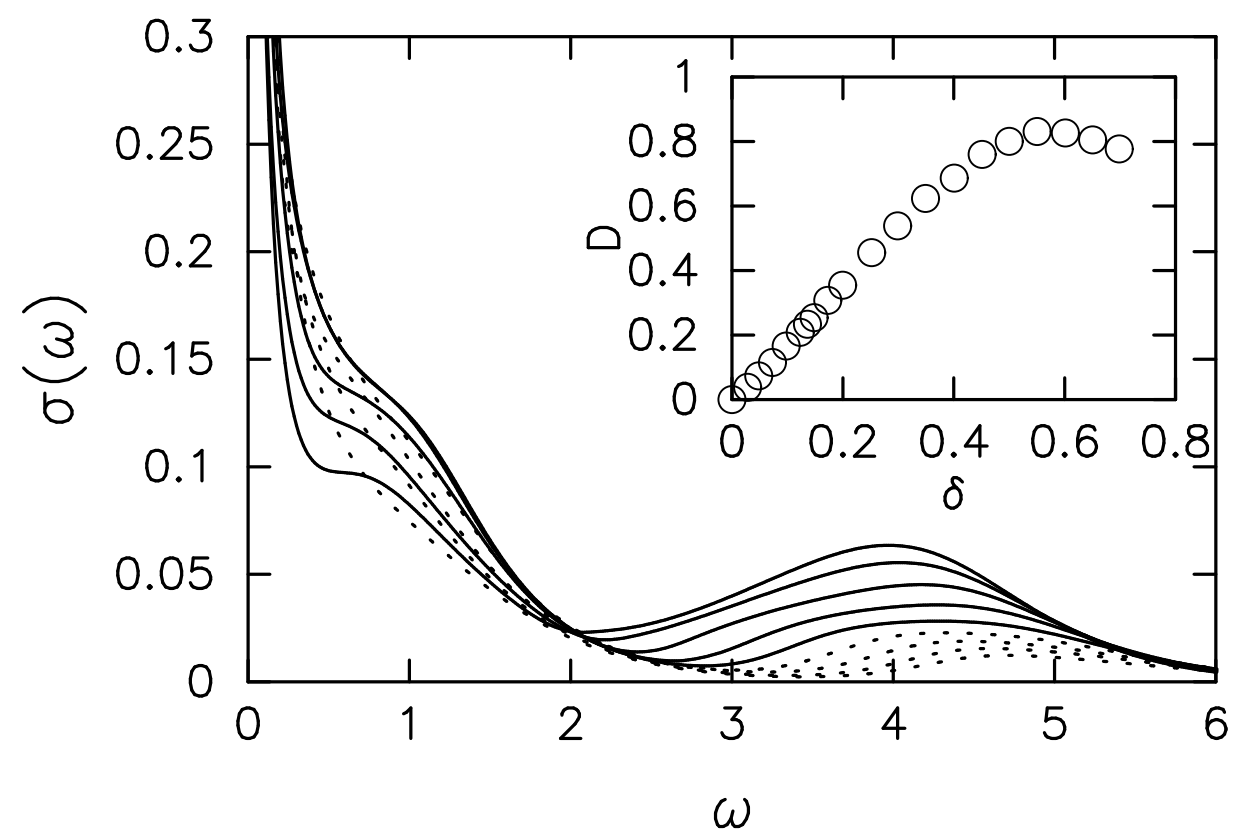

Jarrell, Freericks, and Pruschke, Phys. Rev. B, Figure 2

FIG. 2. The doping dependence of the optical conductivity when $U=4$ and $\beta=43.2$. Note that for larger $\delta$ the mid-IR and Drude peak begin to merge, so that the latter is less distinct. The different dopings are identified by their decreasing charge-transfer peaks, respectively $(\delta=0.068$, 0.0928, 0.1358, 0.1878, 0.2455, 0.3, 0.35, 0.4, and 0.45). The solid lines correspond to the case where the optical conductivity increases with doping in the lower-Hubbard-band region of $\omega<2$ $(\delta<0.25)$ and the dotted lines correspond to the case where $\sigma(\omega)$ decreases with doping $(\delta>0.25)$. The inset shows the evolution of the Drude weight $D$ as a function of doping, which is computed using Eq. G at $\beta=20$. For $\delta \lesssim 0.4$, the Drude weight increases linearly with doping. 


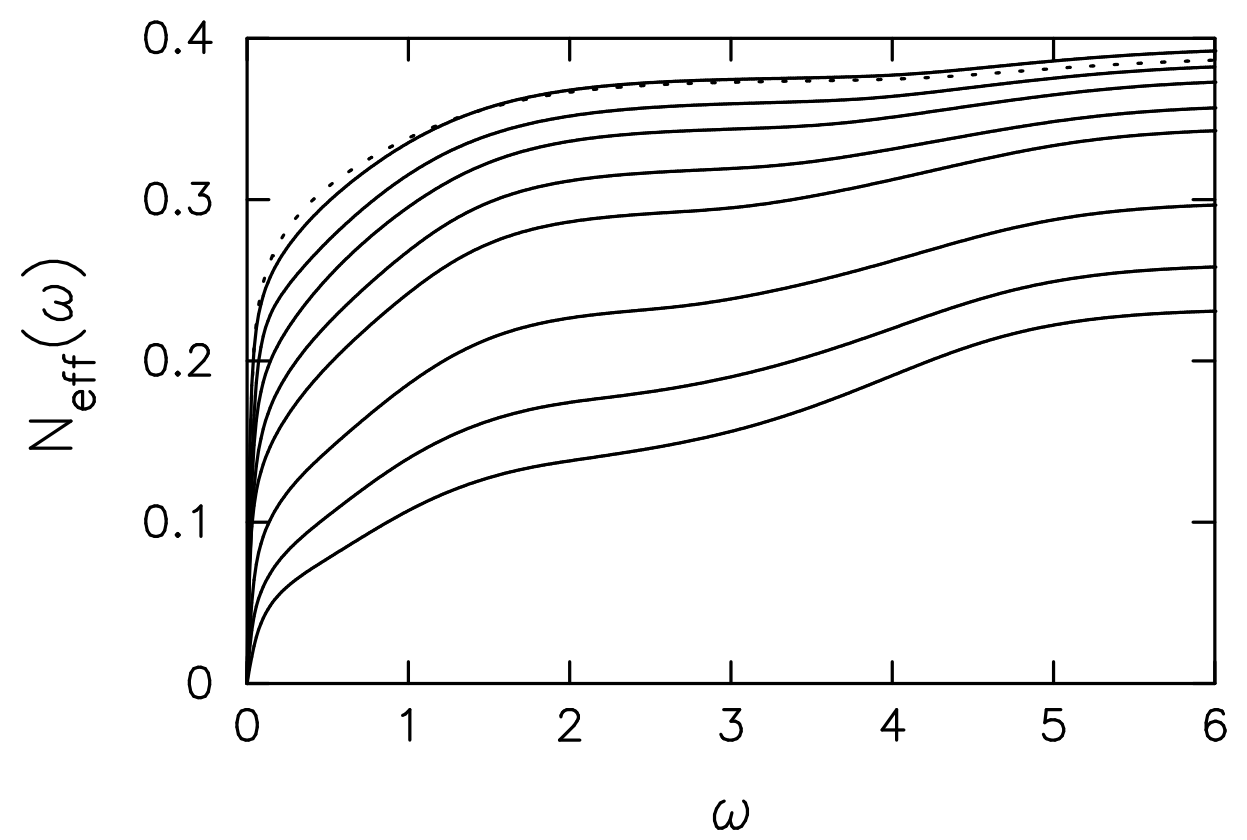

Jarrell, Freericks, and Pruschke, Phys. Rev. B, Figure 3

FIG. 3. Effective charge $N_{\text {eff }}(\omega)$ of the Hubbard model when $U=4$ and $\beta=43.2$ as a function of doping. The same electron concentrations are plotted as in Figure 0 . The solid lines denote doping levels where the effective charge increases with doping, and the dotted line corresponds to the case where the effective charge decreases with doping $(\delta>0.4)$. 


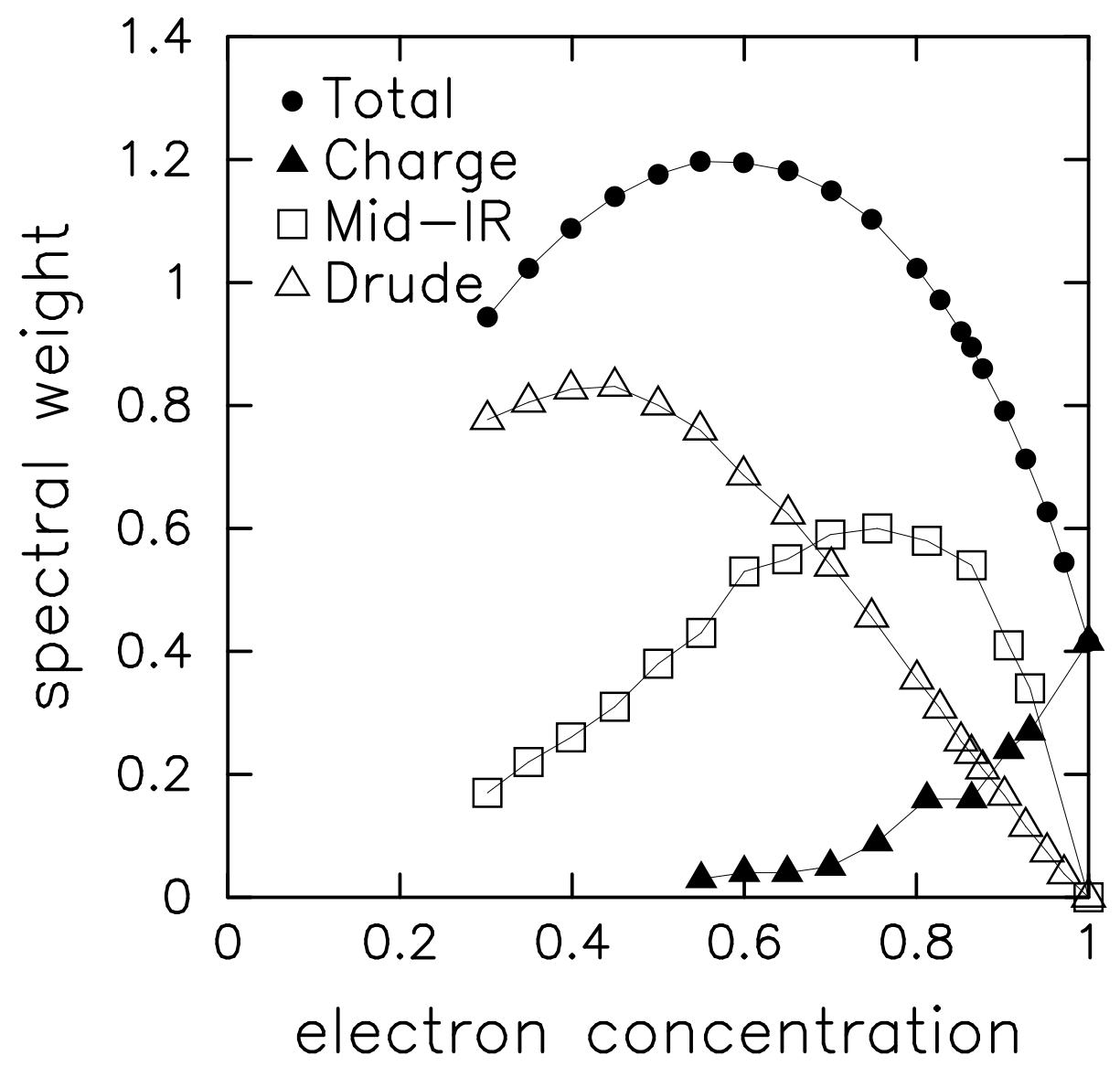

Jarrell, Freericks, and Pruschke, Phys. Rev. B, Figure 4

FIG. 4. Total spectral weight and its individual components for the optical conductivity of the Hubbard model as a function of doping at $U=4$ and $\beta=43.2$. The total spectral weight (solid dots) is broken up into its component pieces (Drude [open triangles], mid-IR [open squares], and charge-transfer [solid triangles]). Note how the charge-transfer peak is important only as the electron concentration nears half filling, and how the mid-IR peak disappears as the electron correlations become smaller. 


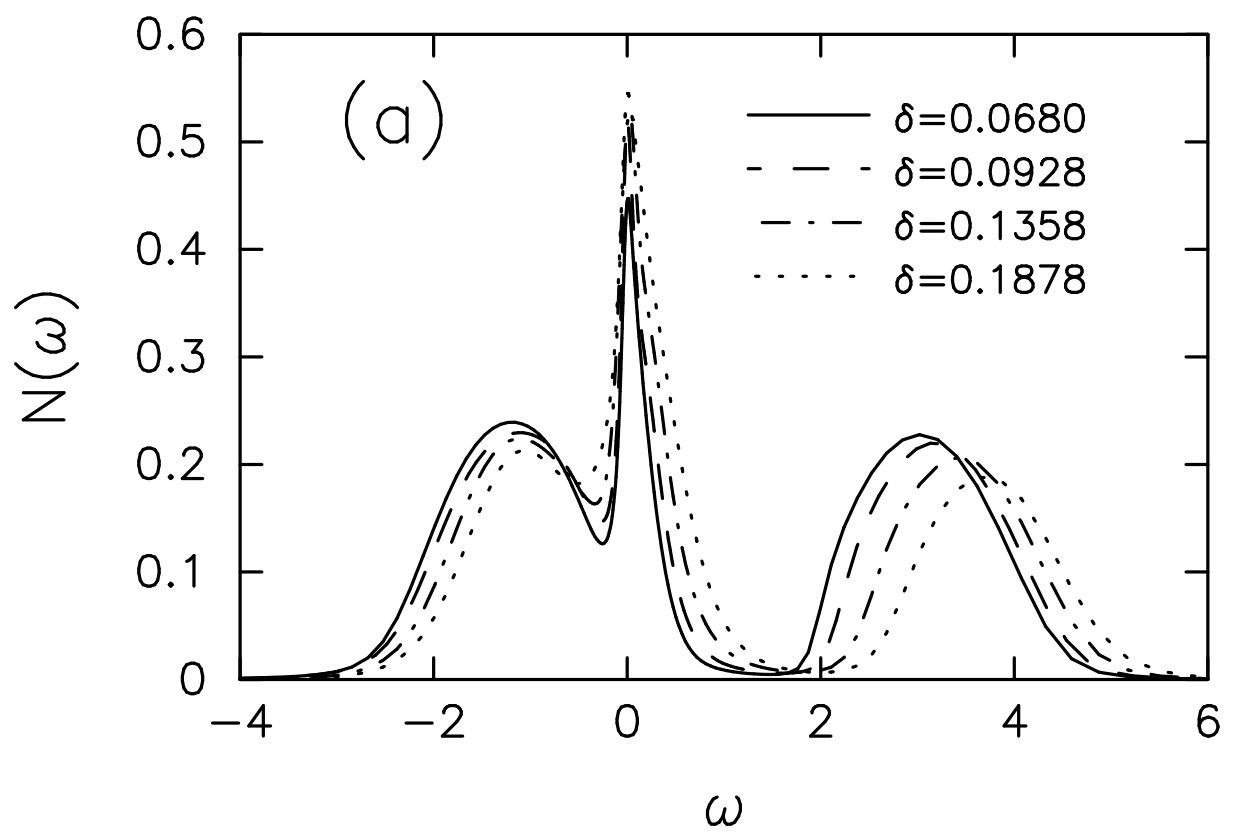

Jarrell, Freericks, and Pruschke, Phys. Rev. B, Figure 5(a)

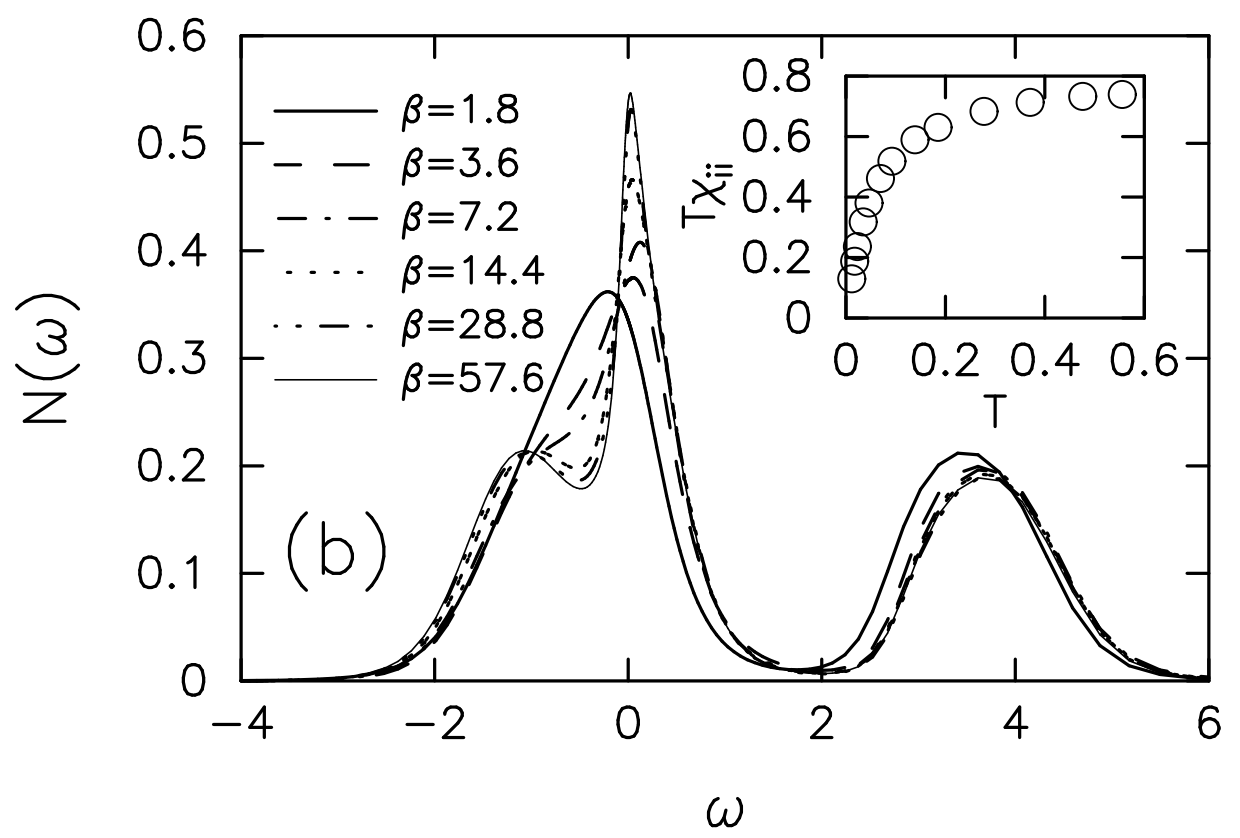

Jarrell, Freericks, and Pruschke, Phys. Rev. B, Figure 5(b) 
FIG. 5. One-particle DOS of the Hubbard model. In (a) the DOS is plotted for different dopings $\delta=1-n$ at $\beta=43.2$. In addition to the lower and upper Hubbard bands, which are fairly doping independent, a resonance occurs at the chemical potential that becomes broader with increasing $\delta$ and finally merges with the lower Hubbard band. In (b), the temperature evolution of the density of states is plotted for $U=4$ and $\delta=0.188$. As the temperature is lowered, a sharp peak, distinct from the lower Hubbard band, develops at the Fermi surface. As shown in the inset, the development of a sharp peak at the Fermi surface is correlated with the reduction of the screened local moment and hence is associated with resonant Kondo screening of the spins. 